\title{
Viability and mitochondrial bioenergetic functions in human colon cancer cells are not affected by treatment with peptides mtCPP1, UPF25, mtgCPP, and mtCPP1gHO
}

Natalja Timohhina ${ }^{1}$, Carmine Pasquale Cerrato $^{2,3}$, Kaido Kurrikoff ${ }^{2}$, Vladimir Chekulayev $^{1}$, Jekaterina Aid-Vanakova ${ }^{1}$, Marju Puurand ${ }^{1}$, Kersti Tepp ${ }^{1}$, Igor Shevchuk ${ }^{1}$, Ulo Langel ${ }^{2}$, Tuuli Kaambre $^{1}$

${ }^{1}$ Laboratory of Chemical Biology, National Institute of Chemical Physics and Biophysics, Akadeemia tee 23, 12618 Tallinn, Estonia

${ }^{2}$ Department of Biochemistry and Biophysics, Stockholm University, Svante Arrhenius väg 16B, 10691 Stockholm, Sweden

${ }^{3}$ Department of Pharmaceutical Sciences, Leslie Dan Faculty of Pharmacy, University of Toronto, Toronto, ON M5S 3M2, Canada

*Corresponding author: Natalja Timohhina, Laboratory of Chemical Biology, National Institute of Chemical Physics and Biophysics, Akadeemia tee 23, 12618 Tallinn, Estonia (e-mail: natalja.timohhina@kbfi.ee) 


\begin{abstract}
The popularity of the specially synthesized cell-penetrating peptides (CPPs) in cancer treatment has grown recently. The main aim of this study was to investigate the effects of four mitochondrially targeted antioxidant CPPs on the viability and bioenergetic function of mitochondria in human adenocarcinoma Caco-2 cells. The number of viable cells was measured by MTT and trypan blue assays. Respirometry and the permeabilized cell technique were applied to measure the mitochondrial function in this cell line. We did not observe any significant effect of CPPs on the mitochondrial reserve respiratory capacity, the function of respiratory chain complexes, and the inclination of these cancer cells to aerobic glycolysis. mtgCPP peptide with the highest antioxidant activity demonstrated improved mitochondrial coupling efficiency. CPPs do not affect mitochondrial function directly but can be considered in therapeutics as a drug-delivery molecule
\end{abstract}

Keywords: cell-penetrating peptide; colorectal cancer; chemotherapy; mitochondria; OXPHOS

Abbreviations used: AK - adenylate kinase; Ap5A - diadenosine pentaphosphate; CAT carboxyatractyloside; CRC colorectal cancer; CPPs - cell-penetrating peptides; HK - hexokinase; FAM - 5-(6)-carboxyfluorescein; MTT - 3-(4,5-dimethylthiazolyl-2)-2, 5diphenyltetrazoliumbromide; OXPHOS - oxidative phosphorylation; ROS - reactive oxygen

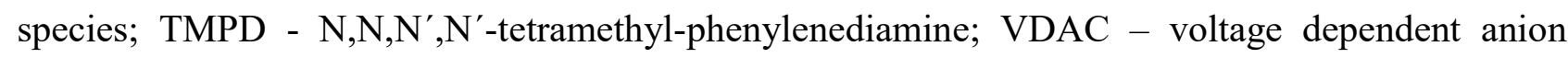
channel.

\title{
1. Introduction
}

Malignant transformation of cells is associated with thorough reprogramming in the metabolic pathways involved in the production of high-energy compounds, as well as in the biosynthesis of cellular lipids, proteins, and nucleic acids [1-3]. Unlike normal cells, cancer cells may metabolize glucose to lactate at high rates even in the presence of oxygen, defined as the Warburg effect $[4,5]$. 
Not all cancer cells rely on glycolysis as their major source of ATP and, because of the oxidative phosphorylation (OXPHOS) system is essential for tumor growth [6-10].

Targeting tumor metabolism is a challenging diagnostic and therapeutic strategy for imaging and selective elimination of cancer cells $[8,11]$. Mitochondria are the promising objects for that due to their specific role in cancer energy metabolism. The targets for the mitochondrial treatment are hexokinase (HK), thiol redox status, VDAC (voltage-dependent anion channel), adenine nucleotide translocase, electron transport chain, mitochondrial inner membrane and tricarboxylic acid cycle [12]. A big challenge is to overcome not so easily penetrable cellular membrane to excess intracellular compartments. Therefore, there is an urgent need for a precise drug-delivery system into mitochondria and the requirement of such a transport mechanism that does not affect the mitochondrial metabolism in an unknown way.

Cell-penetrating peptides (CPPs) are peptides with a sequence length usually between 4 and 30 amino acids. They possess cationic and amphipathic properties, needed characteristics to facilitate the delivery of the cargo molecule. Due to their low cytotoxicity and their possibility to transport many different types of bioactive cargo - such as peptides, proteins, drugs liposomes, phages, plasmid, nucleic acids - they have emerged as a new approach to the treatment of various infection and non-infectious diseases, including cancer [14-18]. Some CPPs can exert a strong antitumor effect or serve as the carriers of polar cytotoxic agents inside malignant cells [14, 19, 20].

Recently, we have synthesized series of mitochondria-targeted (mtCPP), glutathione analog peptides (UPF), and dual antioxidant peptide mtgCPP by fusion of mtCPP1 and UPF25 [21-23] to protect these organelles from oxidative damage $[23,24]$. We have shown that mtCPPs have a superoxide anion scavenging ability in different cell lines, such as HeLa 705 (human cervical carcinoma), bEnd.3 (mouse brain endothelial), U87 (human primary glioblastoma), CHO (Chinese hamster ovary). Even stronger antioxidant activity was detected of the fused peptide mtgCPP compared to mtCPP1 and UPF25 alone [23]. These series of mitochondria targeting peptides also 
exerted a protective effect against exogenously added hydrogen peroxide. The viability of any of the cell lines used in the studies was negatively affected by the treatment with any of these peptides. Colon adenocarcinoma remains the leading cause of morbidity and mortality worldwide. Unlike other types of cancer, colorectal cancer (CRC) partly relies on the mitochondrial OXPHOS as an energy source. Our previous studies showed that CRC cells, unlike many other human cancers, have high rates of basal and ADP-stimulated respiration along with obvious signs of stimulated mitochondrial biogenesis $[9,10]$. Thus, controlling the mitochondrial respiration may be a new strategy for the treatment of CRC [25]. Moreover, it was reported that the intracellular level of ATP is a crucial factor determining chemoresistance in CRC [26]. The influence of the studied CCPs on the mitochondrial function of CPC has not been previously reported. These peptides could suppress the growth of colorectal carcinomas by inhibiting their mitochondrial respiration and/or production of reactive oxygen species (ROS). It is also important to note that recently some specially synthesized peptides have been proposed for the diagnosis of CRC and its treatment as direct cytotoxic agents or carrier molecules [27].

Our study aimed to test the effect of four CPPs with strong antioxidant abilities (mtCPP1, UPF25, mtgCPP, mtCPP1gHO) [23] on the mitochondrial bioenergetic parameters in human CRC cells (Caco-2 line).and to determine the influence of the peptide on the cell viability.

\section{Materials and methods}

\subsection{Chemicals}

Eagle's Minimum Essential Medium (MEM) containing $1.5 \mathrm{~g} / \mathrm{L}$ sodium bicarbonate, non-essential amino acids, L-glutamine, and sodium pyruvate was purchased from Corning (REF 10-009-CVR). Trypsin-EDTA solution, heat-inactivated fetal bovine serum, and antibiotics (penicillin, streptomycin, and gentamicin) were obtained from Gibco Life Technologies (Grand Island, New York, USA). Unless otherwise indicated, all other chemicals were purchased from Sigma-Aldrich. 


\subsection{Peptide synthesis, purification, and analysis}

Solid-phase peptide synthesis, purification, and analysis were performed as described previously [23]. Briefly, peptides were synthesized using standard Fmoc chemistry on microwave-assisted synthesizer (Biotage, Sweden). The peptides were cleaved from the resin, precipitated in ether, and lyophilized. CPPs were also labeled with 5-(6)-carboxyfluorescein (FAM) to obtain FAM-mtCPP1, FAM-UPF25, FAM-mtgCPP, and FAM-mtCPP1gHO (molecular weight: 983.97, 842.5, 1449.5, and 2420.3, respectively) for microscopy studies. The peptides were purified using RP-HPLC on a preparative $\mathrm{C} 8$ column with $\mathrm{H}_{2} \mathrm{O}$-acetonitrile (both solution containing $0.1 \%$ TFA) gradient. The identity of the final products was confirmed using UHPLC-MS (Agilent 1260 Infinity, Agilent Technologies, Santa Clara, California, USA). Stock solutions of the studied peptides (at final concentrations of 0.1 and $1 \mathrm{mM}$ ) were prepared in sterile mQ-water and stored at $-20{ }^{\circ} \mathrm{C}$.

\subsection{Cells}

Caco-2 cells were obtained from the American Type Culture Collection (HTB37TM). These cells were grown in T75 flasks (Greiner Bio-One) in MEM supplemented with 10\% FBS, 100 units/ml penicillin, $100 \mu \mathrm{g} / \mathrm{ml}$ streptomycin, and $50 \mu \mathrm{g} / \mathrm{ml}$ gentamicin at $37{ }^{\circ} \mathrm{C}$ in an incubator with $5 \% \mathrm{CO}_{2}$ in the air. Cells were harvested by mild trypsinization followed by centrifugation at $150 \mathrm{~g}$ for $7 \mathrm{~min}$. The cell pellet was suspended in medium-B [25] supplemented with $2 \mathrm{mg} / \mathrm{mL}$ fatty-acid free bovine serum albumin and $5 \mu \mathrm{M}$ leupeptin (a protease inhibitor) and stored on ice. The viability of these cells was around 95\% according to trypan blue (TB) exclusion test.

Cells were treated with CPPs at $5 \mu \mathrm{M}$ concentrations for $24 \mathrm{hrs}$ at $37{ }^{\circ} \mathrm{C}$, and $5 \% \mathrm{CO}_{2}$ in the air before every assay.

\subsection{Viability assays}

Along with the TB test, cell viability was estimated by MTT Cell Proliferation Assay Kit (ATCC®) 30-1010 K). Caco-2 cells were seeded into Falcon ${ }^{\circledR}$ 96-well microplates (from Corning) with a flat bottom at a density of 5000 cells/well and cultured at $37{ }^{\circ} \mathrm{C}$ with $5 \% \mathrm{CO}_{2}$ for $24 \mathrm{~h}$. After that growth 
medium was replaced with new media containing different CPPs, and cells were incubated overnight. Under the same conditions, growth medium supplemented with sterile milli-Q water was used as a control. The optical density in each well was measured at $570 \mathrm{~nm}$ using $630 \mathrm{~nm}$ as reference wavelength on a FLUOstar Omega (BMG LABTECH, Germany) microplate reader.

\subsection{Assessment of the mitochondrial respiration in Caco-2 cells}

The mitochondrial respiratory activity in Caco-2 cells was evaluated using the saponinpermeabilized cell technique [28].

$\mathrm{O}_{2}$ consumption rate was measured at $25{ }^{\circ} \mathrm{C}$ using a high-resolution Oxygraph-2K respirometer (Oroboros Instruments, Austria); the solubility of oxygen was taken as $240 \mathrm{nmol} / \mathrm{mL}$ [29]. Respiration was measured as a specific oxygen flux (in nmoles $\mathrm{O}_{2} / \mathrm{min} / \mathrm{million}$ cells) and normalized per mg of cellular protein. The protein concentration in cell lysates was determined using the Pierce BCA Protein Kit. The coupling of hexokinase (HK)-catalyzed processes with the OXPHOS system in permeabilized Caco-2 cells was assayed by oxygraphy via activation of mitochondrial respiration by locally generated $\operatorname{ADP}[10,30]$. This effect of glucose on mitochondrial respiration was expressed by glucose index $\left(\mathrm{I}_{\mathrm{GLU}}\right)$ that was calculated according to the equation (1):

$$
I_{G L U}=\frac{V_{G L U}-V_{A T P}}{V_{A D P}-V_{A T P}} \times 100 \%
$$

where $\mathrm{V}_{\mathrm{ADP}}$ is the rate of $\mathrm{O}_{2}$ consumption in the presence of $2 \mathrm{mM}$ ADP, $\mathrm{V}_{\mathrm{GLU}}$ is respiration rate with $10 \mathrm{mM}$ glucose, and $\mathrm{V}_{\mathrm{ATP}}$ is respiration rate upon addition of $0.1 \mathrm{mM}$ ATP. This index indicates the degree to which glucose-mediated stimulation of mitochondrial respiration is comparable to maximal ADP-activated rates of $\mathrm{O}_{2}$ consumption.

The coupling of adenylate (AK)-catalyzed processes with OXPHOS was evaluated by respirometry as described previously $[10,30]$. AK index $\left(\mathrm{I}_{\mathrm{AK}}\right)$ determines the magnitude of this efficiency and is calculated according to the equation (2):

$$
I_{A K}=\frac{\left(V_{A M P}-V_{A p 5 A}\right)}{V_{A p 5 A}}
$$


where $\mathrm{V}_{\mathrm{AMP}}$ and $\mathrm{V}_{\mathrm{Ap} 5 \mathrm{~A}}$ are rates of $\mathrm{O}_{2}$ consumption in the presence of $2 \mathrm{mM}$ AMP and $0.2 \mathrm{mM}$ diadenosine pentaphosphate (Ap5A, an inhibitor of AK), respectively.

\subsection{Statistical Analysis}

All data are expressed as mean values \pm standard error of the mean (SEM) of at least 4-5 independent experiments. Statistical significance was assessed by one-way ANOVA followed by Holm-Sidak post-hoc test using SigmaPlot 11.0 software.

\section{Results and discussion}

\subsection{Toxicity of CPPs on Caco-2 cells}

To evaluate the cytotoxic activity of four different CPPs against human epithelial colorectal adenocarcinoma cells (Caco-2) were incubated with a dose of $5 \mu \mathrm{M}$ for $24 \mathrm{~h}$. Cell viability was determined by the TB exclusion test and MTT assay. Studied FAM-labeled CPPs did not affect the Caco-2 cells proliferation rate no difference was observed between control and CPP-treated cells (Fig. 1). Similar effects were observed with HeLa 705, U87, and bEnd.3 cell lines as reported earlier $[23,31]$.

\subsection{Effect of CPPs on the coupling of OXPHOS with hexokinase-and adenylate kinase-catalyzed} processes in Caco-2 cells

Human CRC cells compared with unaffected tissue cells have an increased energy demand, which is manifested in their increased glycolytic activity and rates of OXPHOS [9, 25]. Colorectal adenocarcinoma maintains high rates of glucose consumption and has a higher tendency to aerobic glycolysis [9, 32]. This phenomenon may be related to the elevated expression of some glucose transporters [33] and glycolytic enzymes [34, 35], or predominantly, by the mitochondrial VDACbound HK-1 or 2, which use mitochondrially generated ATP to phosphorylate glucose in large amounts $[5,9,10]$. In this work, we evaluated Caco-2 cells as a model system expressing VDACassociated HK-2 similarly to human colorectal cancer [25]. Experiments were carried out to estimate the impact of CPPs on the inclination of Caco-2 cells to aerobic glycolysis (the Warburg 
behavior). The addition of $10 \mathrm{mM}$ glucose to permeabilized cells caused an increase in the respiration rate in both the CPP-treated and control cells but there is no significant difference between those groups (Fig. 2). Thus, the close coupling between the HK and OXPHOS system was maintained in all experiments. The effect of glucose consumption was expressed by the glucose index that reflects the degree of glucose response relative to the maximal rate of ADP-activated respiration $\mathrm{I}_{\mathrm{GLU}}$. The measured $\mathrm{HK}$ index value for control cells $(12.2 \%)$ was very similar to the values measured for the peptide-treated cells (Table 1). The ADP activated respiration was inhibited by the addition of an inhibitor of adenine nucleotide translocator, carboxyatractyloside, which demonstrates the complete intactness of the mitochondrial inner membrane. These data confirm that all four peptides did not affect the Warburg behavior of Caco-2 cells.

The AK-catalyzed phosphotransfer system plays a key role in the maintenance of energy homeostasis in well-differentiated cells with intermittent high-energy demand, such as neural, cardiac, and skeletal muscle cells [36]. It is upregulated in human CRC [37] with the total AK activity about 2 times higher than surrounding normal tissue [10]. The same was also observed in tumor cells of other histological types [38]. The AK system could play an important role in the adaptation of cancer cells to an unfavorable microenvironment and tumor progression [39, 40]. Taking into account the role of AK regulated processes in tumor growth we evaluated the effect of CPPs on of this system in Caco-2 cells through its coupling with OXPHOS. Addition of AMP (in the presence of $0.1 \mathrm{mM}$ ATP) to permeabilized cells leads to nearly $30 \%$ increase in the rate of $\mathrm{O}_{2}$ consumption (Fig. 3). Ap5A suppressed the AMP-stimulated respiration, indicating thereby that it was largely mediated by ADP realized in AK reactions. AK index describes the functional activity of AK processes and their coupling with the OXPHOS system. All CPPs did not affect the function of AK system, since the value of $\mathrm{I}_{\mathrm{AK}}$ measured for control cells $(0.50 \pm 0.16)$ did not statistically differ from the value measured for peptide-treated cells ( Table 1). 


\subsection{Effect of CPPs on the functional activity of mitochondrial respiratory complexes in Caco-2 cells and the reserve respiratory capacity of these organelles}

Previous studies showed that all the studied CPPs can internalize into human cervical carcinoma cells and some other cell lines $[23,31]$. Also, mtgCPP and mtCPP1 have the increased ability to target mitochondria without any effect on mitochondrial membrane potential and ATP production by these cells [23]. We proposed that these peptides could affect the bioenergetic function of mitochondria with the elevated OXPHOS activity in CRC cells, unlike glycolytic cervical carcinomas [41, 42] 10, 25, 43].

To investigate the influence of FAM-labeled mtCPP1, UPF25, mtgCPP, and CPP1gHO peptides on the oxygen consumption of the CRC cells and the mitochondrial respiratory system enzymes, we used high-resolution respirometry protocol described previously [44].Mitochondrial respiration in permeabilized Caco-2 cells in the presence of malate and glutamate (LEAK) almost doubled after the addition of $2 \mathrm{mM}$ ADP, indicating the active state of Complex I (Fig. 4A). Succinate (final concentration $10 \mathrm{mM}$ ) abrogated the strong inhibitory effect of rotenone and exceeded the Complex I maximal rate, showing that the mitochondrial Complex II is more active versus Complex I (Table 2). The succinate-fueled respiration was suppressed by the addition of $10 \mu \mathrm{M}$ antimycin-A, inhibiting the electron flow from Complex III to cytochrome c. Mitochondrial Complex IV was activated by $1.0 \mathrm{mM}$ TMPD, the artificial substrate for reducing cytochrome $\mathrm{c}$ in the presence of 5 $\mathrm{mM}$ ascorbate, which also shows the total capacity of the respiratory chain (Fig. 4A). Finally, the TMPD-activated respiration was inhibited by the addition of $1 \mathrm{mM} \mathrm{NaCN}$, completely inhibiting Complex IV. The mitochondrial respiratory system is the main generator of ROS [45], associated in signaling with cellular stress responses, cell proliferation, and apoptosis [46]. In murine and human tumor models, two different events, mitochondrial respiratory chain overload and partial electron transport inhibition promote superoxide-dependent tumor cell migration, invasion, and metastasis $[47,48]$. We found that none of the tested peptides had any effect on the activities of individual respiratory complexes as compared to control cells (Fig.4B). However, in the presence of UPF25 
Complex II-linked respiration is significantly higher than mtgCPP (Fig. 4B). Besides, a significant increase in the respiratory control ratio (RCR) was detected after treatment with mtgCPP The indicator of the coupling state of mitochondria was calculated as the ratio of maximal ADPstimulated respiration in the presence of Complex I-linked substrates (glutamate and malate) and the LEAK component. These results indicate a tighter coupling of electron transport and efficient OXPHOS in the CaCo-2 cells treated with the dual antioxidant peptide mtgCPP.

The maximal mitochondrial respiration was measured in the uncoupled state induced by gradual titration with FCCP (p-(trifluoro-methoxy)phenyl-hydrazone) of the untreated and CPP-treated Caco-2 cells according to previously described protocols [49] (Fig. 5A). FCCP is a protonophore that uncouples electron transport and mitochondrial respiration from ATP synthesis by disrupting the proton gradient. Respiration rates of control and CPPs treated Caco- 2 cells increased about 3 times compared with the proton leak. The optimal concentration of FCCP for peptide treated and untreated cells were $2.21 \pm 0.19 \mu \mathrm{M}$ since at higher concentrations of this uncoupler a decrease in the rate of oxygen consumption was observed. Finally, the electron transport was inhibited by $2.5 \mu \mathrm{M}$ rotenone and $10 \mu \mathrm{M}$ antimycin, a Complex III inhibitor, to subtract the non-mitochondrial oxygen consumption. Proton leak and ATP-linked respiration rate are the mitochondrial coupling efficiency indicators. Proton leak is assessed under the condition of the oligomycin - suppressed ATP synthase. None of the peptides tested had any effect of the coupling between ATP synthesis and OXPHOS (Fig. 5B). Mitochondrial reserve capacity is the spare ATP amount produced by the mitochondrial respiratory system in case of cell stress or high-energy demand. The summary of the respiratory values had not revealed any alterations (Fig. 5B). Statistical analyses confirmed no difference between experimental groups.

\section{Conclusion}

None of the tested peptides had any effect on the viability of Caco-2 cells, as well as on their mitochondrial reserve respiratory capacity, the function of respiratory chain complexes, and the small inclination of these cancer cells to aerobic glycolysis. Patients with high levels of 
mitochondrial markers could be treated with mitochondrial-based therapies in addition to the standard cure to effectively prevent tumor recurrence, metastasis, and drug-resistance [50]. Due to their antioxidative properties and having no negative impact on mitochondrial bioenergetics these mitochondria-targeting CCPs, if conjugated with some specific metabolic regulator for diseased cells, can serve as carrier molecules safe for non-diseased cells. Anti-cancer agents, called "mitocans", could serve as potential cargo molecules [51] for the studied peptides, which would allow precise delivery of the medication.

In addition to the cancer treatment, these peptides might have potential therapeutic applications in pathologies caused by mitochondrial dysfunctions and aging.

\section{Funding}

This work was supported by the EU through the European Regional Development Fund CoE program TK133, the Swedish Research Council (VR-NT), and the Swedish Cancer Foundation.

\section{Conflicts of interest}

The authors declare no conflict of interest.

\section{Tables and figure legends}

Table 1. Effects of cell-penetrating FAM-labeled peptides on the coupling of AK-and HK-catalyzed processes with OXPHOS in Caco-2 cells

\begin{tabular}{|l|c|c|c|c|c|}
\hline Parameters & control & mtCPP1 & UPF25 & mtgCPP & mtCPPgHO \\
\hline AK index & $0.50 \pm 0.16$ & $0.60 \pm 0.15$ & $0.56 \pm 0.19$ & $0.53 \pm 0.07$ & $0.58 \pm 0.15$ \\
\hline HK index, \% & $12.2 \pm 1.5$ & $13.7 \pm 2.4$ & $12.2 \pm 2.1$ & $13.6 \pm 1.3$ & $11.3 \pm 2.0$ \\
\hline
\end{tabular}

Data are shown as mean values \pm SEM, $n=4-5$. 
Table 2. The influence of FAM-labeled CPPS on the ratio of respiratory Complex I and II in mitochondrial activity

\begin{tabular}{|l|c|c|c|c|c|}
\hline \multirow{2}{*}{ Oarameters of } & \multicolumn{5}{|c|}{ Caco-2 cells } \\
\cline { 2 - 6 } & control & mtCPP1 & UPF25 & mtgCPP & mtCPP1gHO \\
\hline Vo & $1.08 \pm 0.06$ & $1.04 \pm 0.06$ & $1.18 \pm 0.14$ & $0.9 \pm 0.05$ & $1.18 \pm 0.07$ \\
\hline $\mathrm{V}_{\mathrm{ADP}}$ & $2.07 \pm 0.09$ & $1.99 \pm 0.16$ & $2.30 \pm 0.19$ & $1.94 \pm 0.18$ & $2.11 \pm 0.08$ \\
\hline $\mathrm{V}_{\text {Rot }}$ & $0.46 \pm 0.02$ & $0.38 \pm 0.05$ & $0.53 \pm 0.05$ & $0.43 \pm 0.06$ & $0.47 \pm 0.04$ \\
\hline $\mathrm{V}_{\text {Suc }}$ & $2.64 \pm 0.11$ & $2.34 \pm 0.29$ & $3.05 \pm 0.21$ & $2.40 \pm 0.24$ & $2.65 \pm 0.16$ \\
\hline $\mathrm{CI} / \mathrm{CII}$ & $\mathbf{0 . 7 4}$ & $\mathbf{0 . 8 2}$ & $\mathbf{0 . 7 0}$ & $\mathbf{0 . 7 7}$ & $\mathbf{0 . 7 5}$ \\
\hline
\end{tabular}

Each data point representing rates of $\mathrm{O}_{2}$ consumption is a mean value $\pm \mathrm{SEM}, \mathrm{n}=4$; values are expressed in nmoles $\mathrm{O}_{2} /$ min per mg of cell protein and are obtained according to the experimental data shown in Fig. 4A; Vo is LEAK respiration in the presence of $5 \mathrm{mM}$ glutamate and $2 \mathrm{mM}$ malate, Complex I-linked substrates; $\mathrm{V}_{\mathrm{ADP}}-2 \mathrm{mM}$ ADP-stimulated respiration; $\mathrm{V}_{\mathrm{Rot}}-$ rates of $\mathrm{O}_{2}$ consumption in the presence of $2.5 \mu \mathrm{M}$ rotenone (an inhibitor of Complex I); $\mathrm{V}_{\text {Suc }}$ - respiration rate in the presence of $10 \mathrm{mM}$ succinate and $2.5 \mu \mathrm{M}$ rotenone, $\mathrm{CI} / \mathrm{CII}-$ Complex I and Complex II respiration rate ratio.

Figure legends

\section{(A)}

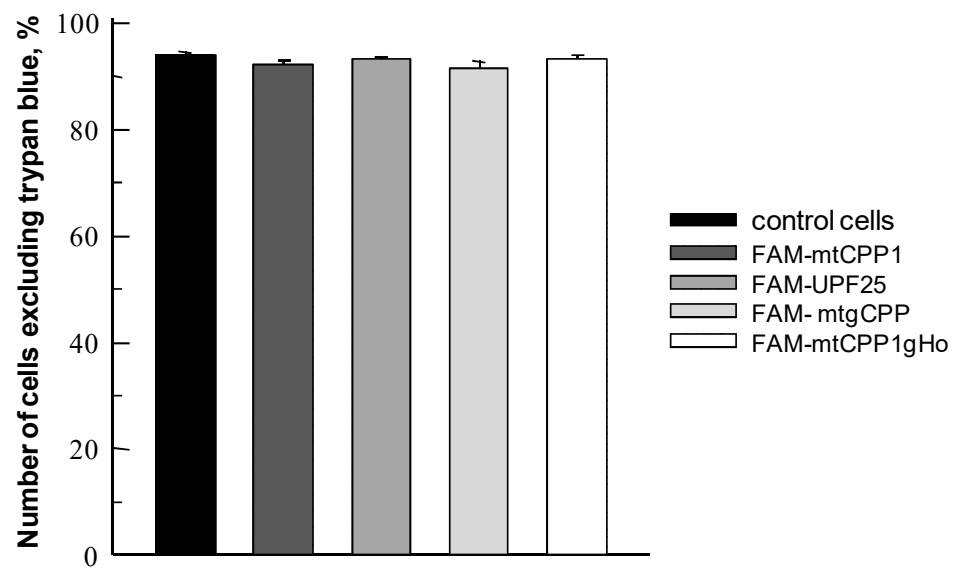

(B)

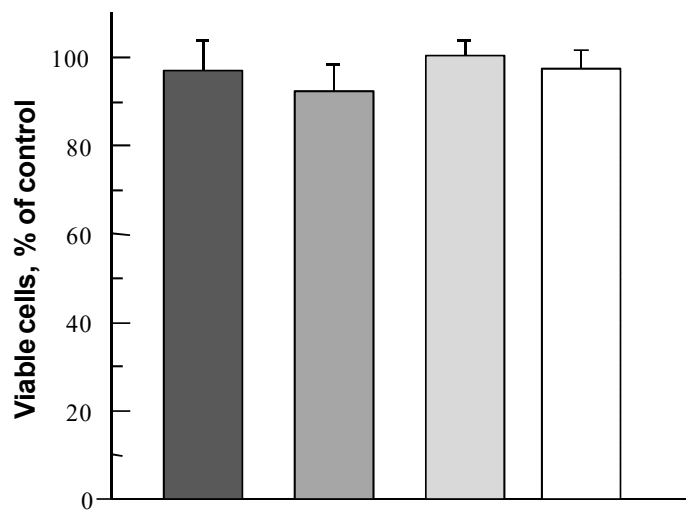


Figure 1. The effect of cell-penetrating FAM-labeled peptides at final concentrations of $5 \mu \mathrm{M}$ on the viability of Caco-2 cells in trypan blue exclusion assay (A) and their proliferative activity that was estimated by MTT test (B). Cells were treated with the indicated peptides in complete growth medium for $24 \mathrm{hrs}$ at $37^{\circ} \mathrm{C}$. Data are shown as mean values \pm SEM, $\mathrm{n}=4$.

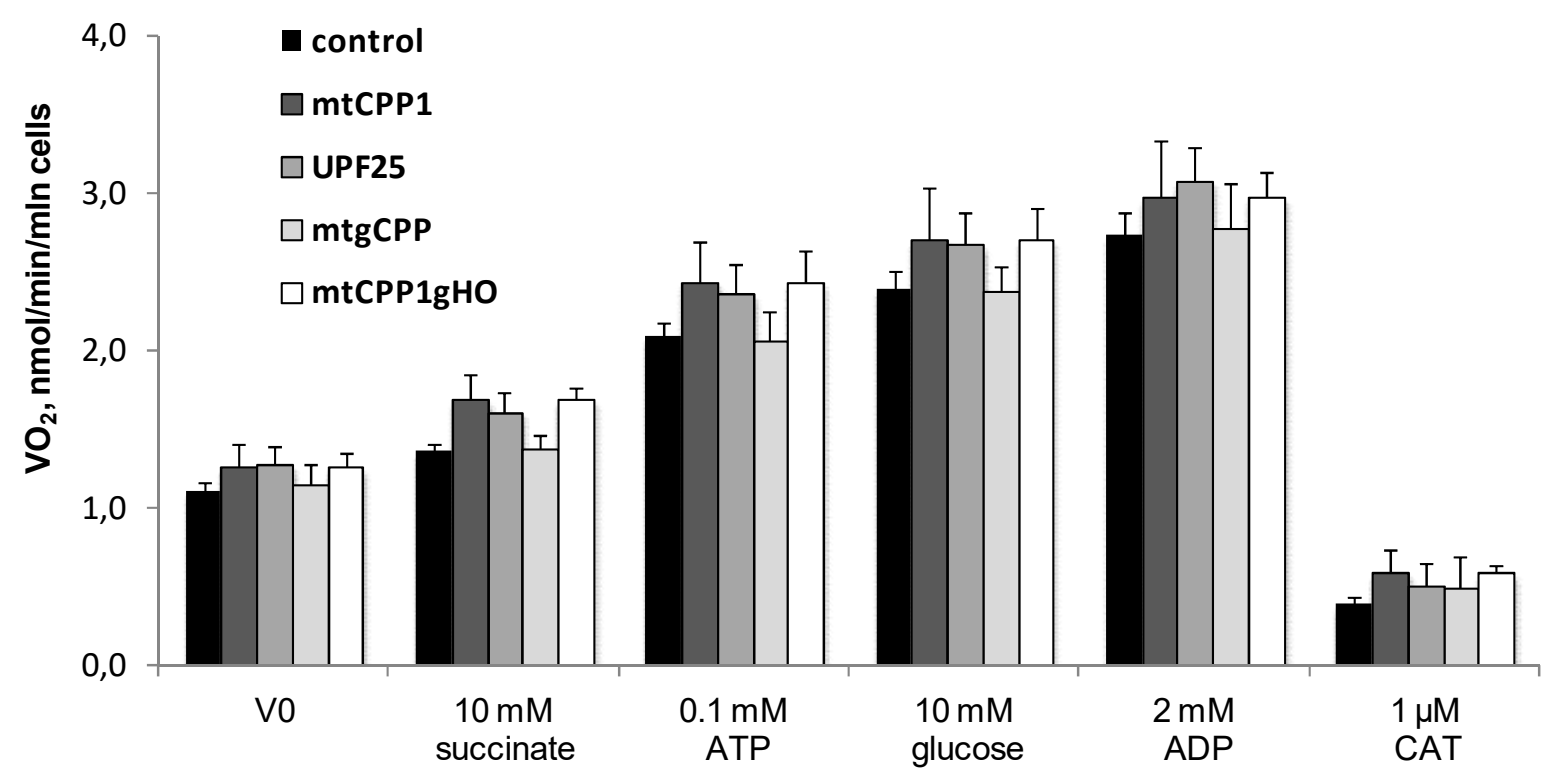

Figure 2. Oxygraphic analysis of the coupling between hexokinase -catalyzed processes and OXPHOS in control and CPPs treated at $5 \mu \mathrm{M}$ for $24 \mathrm{hrs}$ Caco-2 cells; these experiments were carried out in medium-B with $5 \mathrm{mM}$ glutamate and $2 \mathrm{mM}$ malate as the respiratory substrates. Succinate, glucose, the corresponding adenine nucleotides (ATP or ADP), and carboxyatractyloside (CAT, an inhibitor of adenine nucleotide translocator) were added to the cells sequentially as indicated on the x-axis. VO2 means the rate of oxygen consumption. Data are shown as mean values $\pm \mathrm{SEM}, \mathrm{n}=5$. 


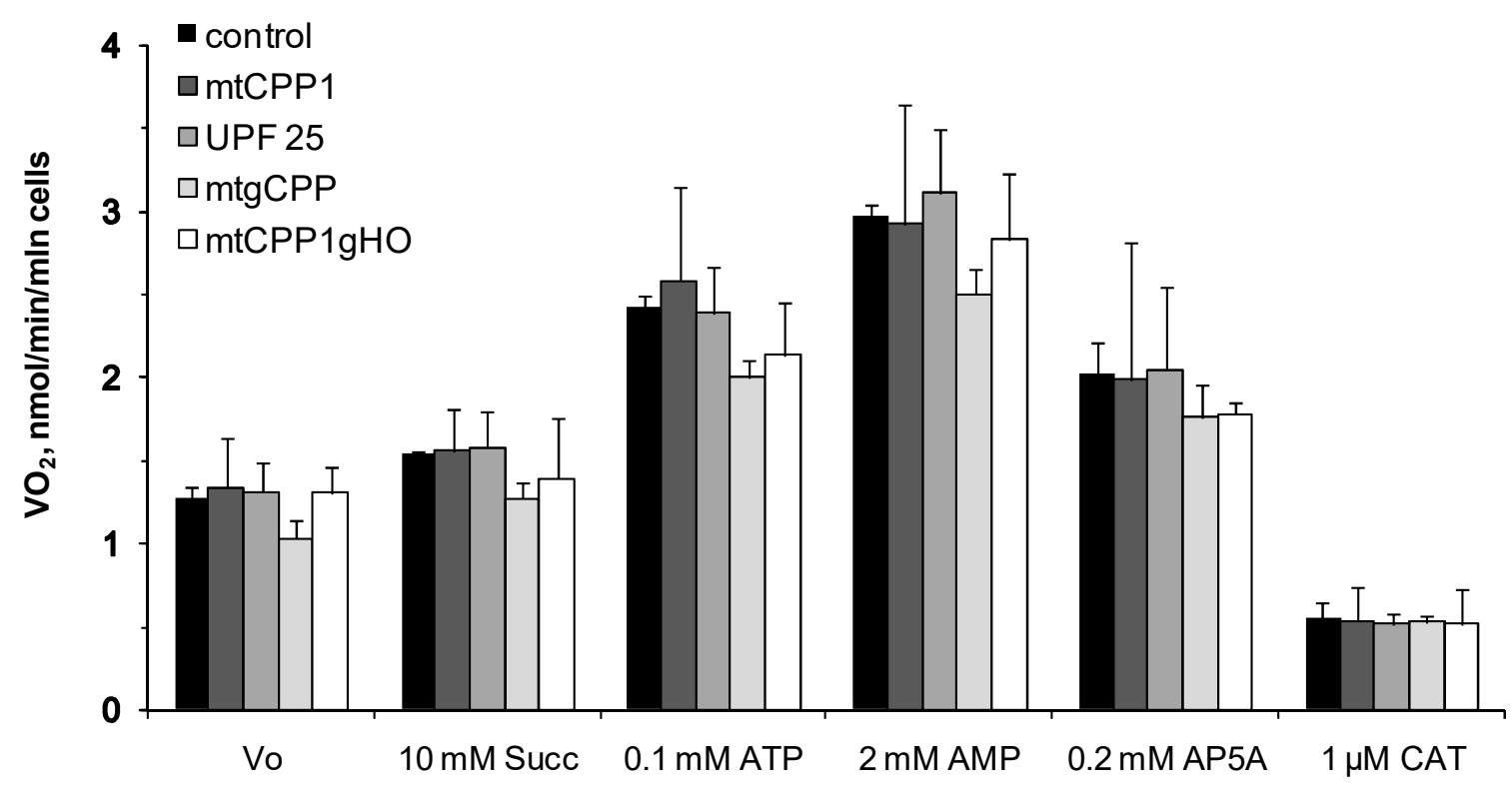

Figure 3. Oxygraphic analysis of the functional coupling between adenylate kinase (AK) catalyzed processes and OXPHOS in control and CPPs pre-treated Caco-2 cells at $5 \mu \mathrm{M}$ for $24 \mathrm{hrs}$; addition of $2 \mathrm{mM}$ AMP in the presence of $0.1 \mathrm{mM}$ ATP resulted in activation of mitochondrial respiration due to formation of ADP in AK reactions. The involvement of $\mathrm{AK}(\mathrm{s})$ in the stimulation of mitochondrial respiration was confirmed by subsequent addition of diadenosine pentaphosphate (Ap5A), an inhibitor of AK; as can be seen, the addition of Ap5A caused a strong decrease in the rate of $\mathrm{O}_{2}$ consumption by these tumor cells. Carboxyatractyloside (CAT) - a selective inhibitor of adenine nucleotide carrier was added to control the intactness of the mitochondrial inner membrane. Data are shown as mean values \pm SEM, $n=4-6$. 


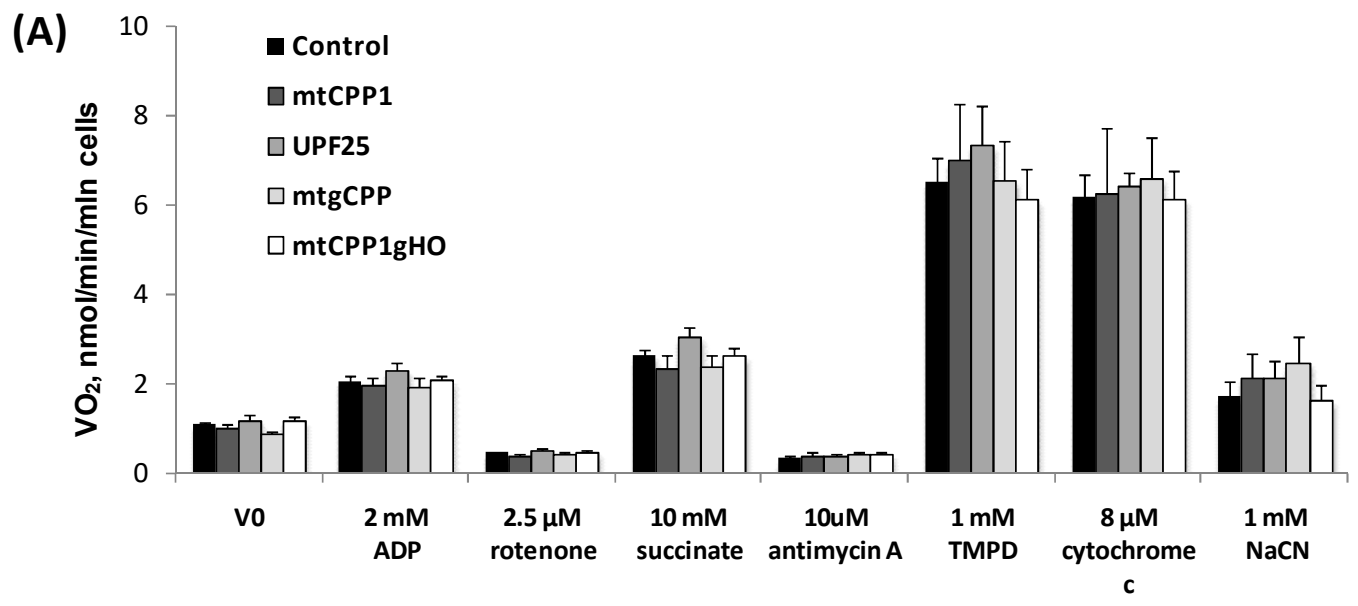

(B)
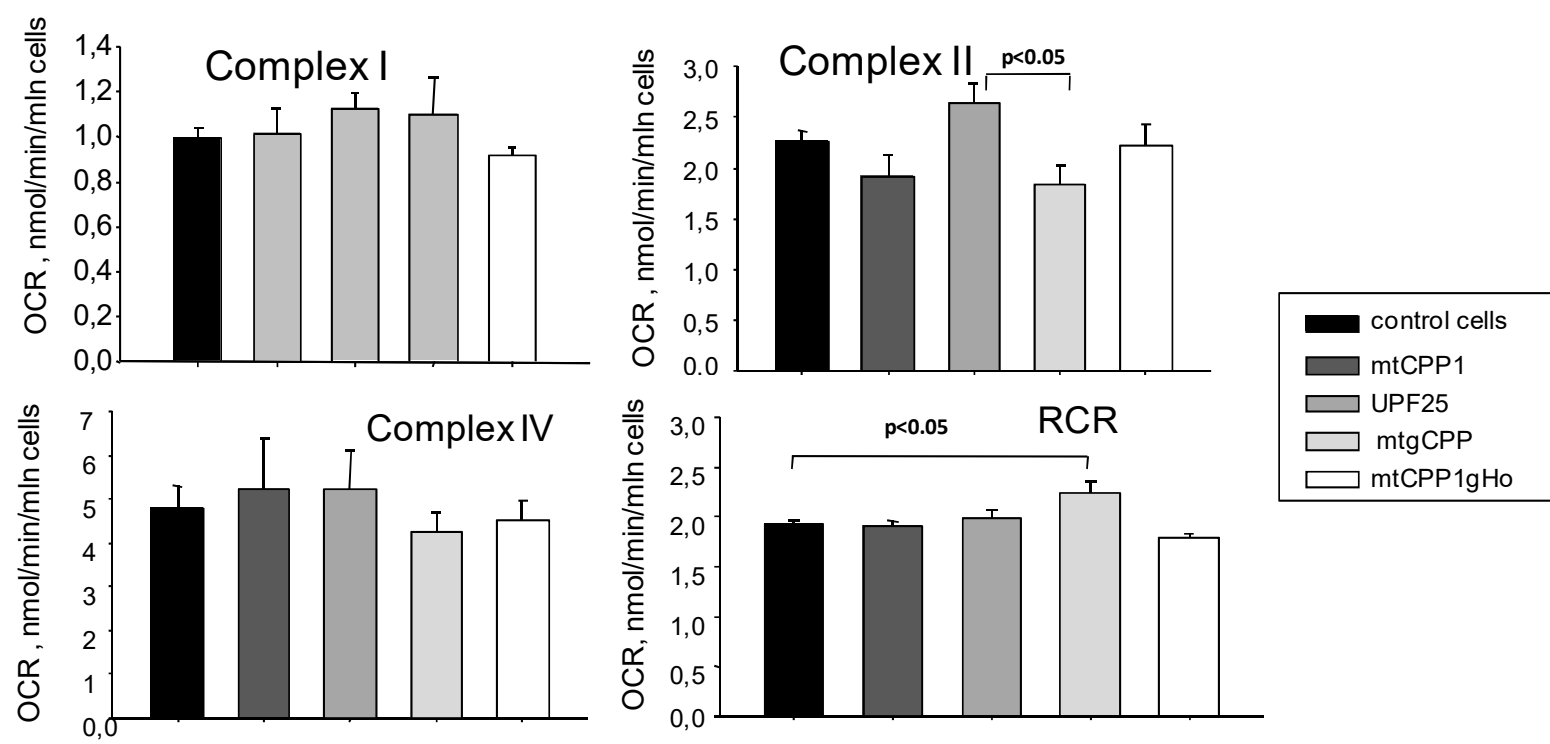

Figure 4. Effect of various CPPs on respiration of the permeabilized Caco-2 cells (A)

Mitochondrial OXPHOS function test was followed a sequential activation-inhibition of Complexes I-IV of the electron transport system. Oxygen consumption rates expressed as respiration per million cells (nmol/min/mln cells) (B) One-way ANOVA indicated no significant differences between mitochondrial respiratory Complexes I, II, IV activities in the CPP-treated and control groups. The respiratory control ratio (RCR) is a measure of mitochondrial coupling state for Complex I. Data are shown as mean values \pm SEM, $n=4$. 
(A)

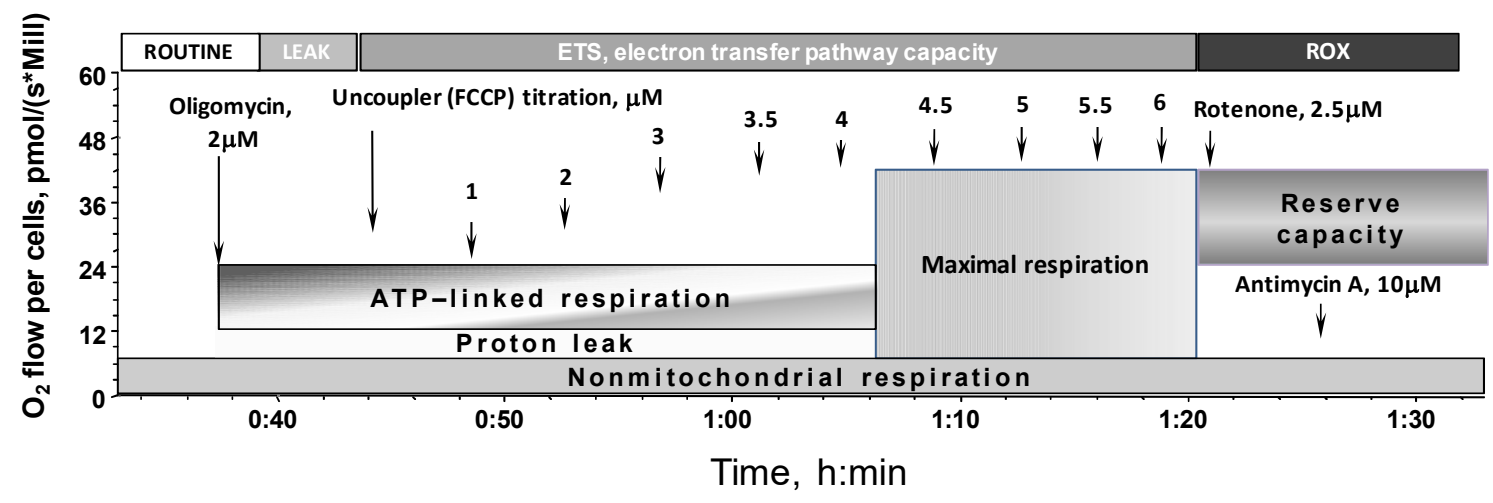

(B)

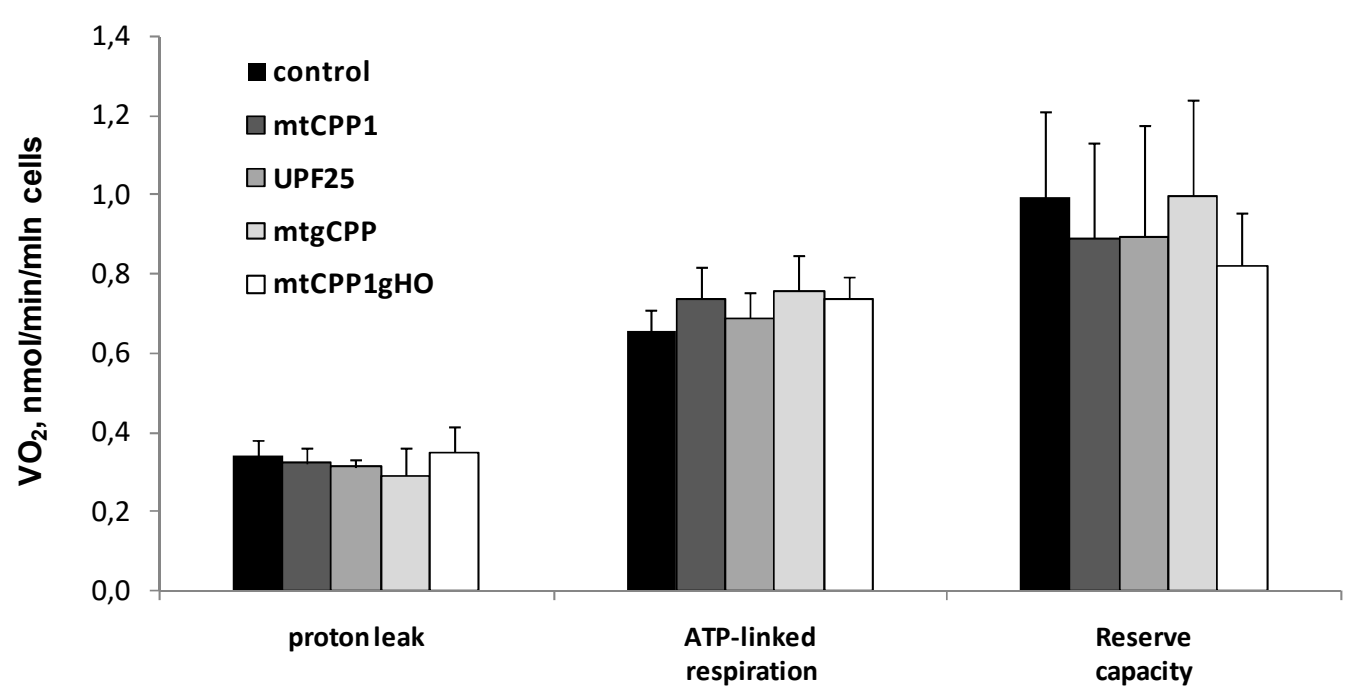

Figure 5. Assessment of mitochondrial function in control and CPP-treated intact Caco-2 cells. (A) Representative respirometric traces with a schematic representation of the used mitochondrial respiratory reserve capacity assay; arrows indicate the FCCP concentrations added into the oxygraph chamber. (C) Calculated functional properties of mitochondrial respiration in control and CPP-treated Caco-2 cells. These cells were treated with the indicated peptides at a final concentration of $5 \mu \mathrm{M}$ in complete growth medium for $24 \mathrm{hrs}$ at $37^{\circ} \mathrm{C}$. Data are shown as mean values $\pm \mathrm{SEM}, \mathrm{n}=5$.

\section{References}

[1] R.J. DeBerardinis, N.S. Chandel, Fundamentals of cancer metabolism, Science Advances 2(5) (2016) e1600200. 
[2] S. Xiong, Y. Feng, L. Cheng, Cellular Reprogramming as a Therapeutic Target in Cancer, Trends Cell Biol. (2019).

[3] J.K. Byun, M. Park, J.W. Yun, J. Lee, J.S. Kim, S.J. Cho, Y.M. Lee, I.K. Lee, Y.K. Choi, K.G. Park, Oncogenic KRAS signaling activates mTORC1 through COUP-TFII-mediated lactate production, EMBO reports 20(6) (2019) e47451.

[4] T. Fan, G. Sun, X. Sun, L. Zhao, R. Zhong, Y. Peng, Tumor Energy Metabolism and Potential of 3-Bromopyruvate as an Inhibitor of Aerobic Glycolysis: Implications in Tumor Treatment, Cancers (Basel) 11(3) (2019).

[5] S.P. Mathupala, Y.H. Ko, P.L. Pedersen, Hexokinase II: cancer's double-edged sword acting as both facilitator and gatekeeper of malignancy when bound to mitochondria, Oncogene 25(34) (2006) 4777-86.

[6] C. Jose, N. Bellance, R. Rossignol, Choosing between glycolysis and oxidative phosphorylation: A tumor's dilemma?, Biochimica et Biophysica Acta (BBA) - Bioenergetics 1807(6) (2011) 552561.

[7] N.D. Amoedo, M.F. Rodrigues, F.D. Rumjanek, Mitochondria: are mitochondria accessory to metastasis?, Int. J. Biochem. Cell Biol. 51 (2014) 53-7.

[8] Y. Shi, S.K. Lim, Q. Liang, S.V. Iyer, H.-Y. Wang, Z. Wang, X. Xie, D. Sun, Y.-J. Chen, V. Tabar, P. Gutin, N. Williams, J.K. De Brabander, L.F. Parada, Gboxin is an oxidative phosphorylation inhibitor that targets glioblastoma, Nature 567(7748) (2019) 341-346.

[9] A. Kaldma, A. Klepinin, V. Chekulayev, K. Mado, I. Shevchuk, N. Timohhina, K. Tepp, M. Kandashvili, M. Varikmaa, A. Koit, M. Planken, K. Heck, L. Truu, A. Planken, V. Valvere, E. Rebane, T. Kaambre, An in situ study of bioenergetic properties of human colorectal cancer: the regulation of mitochondrial respiration and distribution of flux control among the components of ATP synthasome, Int. J. Biochem. Cell Biol. 55 (2014) 171-86.

[10] V. Chekulayev, K. Mado, I. Shevchuk, A. Koit, A. Kaldma, A. Klepinin, N. Timohhina, K. Tepp, M. Kandashvili, L. Ounpuu, K. Heck, L. Truu, A. Planken, V. Valvere, T. Kaambre, Metabolic remodeling in human colorectal cancer and surrounding tissues: alterations in regulation of mitochondrial respiration and metabolic fluxes, Biochemistry and Biophysics Reports 4 (2015) 111-125.

[11] P.J. Akhenblit, M.D. Pagel, Recent Advances in Targeting Tumor Energy Metabolism with Tumor Acidosis as a Biomarker of Drug Efficacy, Journal of cancer science \& therapy 8(1) (2016) 20-29.

[12] B. Yan, L. Dong, J. Neuzil, Mitochondria: An intriguing target for killing tumour-initiating cells, Mitochondrion 26 (2016) 86-93.

[13] F. Sotgia, B. Ozsvari, M. Fiorillo, E.M. De Francesco, G. Bonuccelli, M.P. Lisanti, A mitochondrial based oncology platform for targeting cancer stem cells (CSCs): MITO-ONC-RX, Cell Cycle 17(17) (2018) 2091-2100.

[14] A.D. Woldetsadik, M.C. Vogel, W.M. Rabeh, M. Magzoub, Hexokinase II-derived cellpenetrating peptide targets mitochondria and triggers apoptosis in cancer cells, The FASEB Journal $31(5)(2017) 2168-2184$. 
[15] H.-K. Kwon, M.C. Patra, H.-J. Shin, X. Gui, A. Achek, S. Panneerselvam, D.-J. Kim, S.-J. Song, R. Hong, K.S. Kim, Y.G. Kim, F.Y. Lee, D.-H. Hahm, S.H. Lee, S. Choi, A cell-penetrating peptide blocks Toll-like receptor-mediated downstream signaling and ameliorates autoimmune and inflammatory diseases in mice, Exp. Mol. Med. 51(4) (2019) 50.

[16] P. Lu, B.J. Bruno, M. Rabenau, C.S. Lim, Delivery of drugs and macromolecules to the mitochondria for cancer therapy, J Control Release 240 (2016) 38-51.

[17] A. Jain, A. Chugh, Mitochondrial transit peptide exhibits cell penetration ability and efficiently delivers macromolecules to mitochondria, FEBS Lett. 590(17) (2016) 2896-905.

[18] S.-k. Yang, A.-m. Li, Y.-c. Han, C.-h. Peng, N. Song, M. Yang, M. Zhan, L.-f. Zeng, P.-a. Song, W. Zhang, S.-q. Tang, H. Zhang, Mitochondria-Targeted Peptide SS31 Attenuates Renal Tubulointerstitial Injury via Inhibiting Mitochondrial Fission in Diabetic Mice, Oxidative Medicine and Cellular Longevity 2019 (2019) 13.

[19] Y.-F. Xiao, M.-M. Jie, B.-S. Li, C.-J. Hu, R. Xie, B. Tang, S.-M. Yang, Peptide-Based Treatment: A Promising Cancer Therapy, Journal of Immunology Research 2015 (2015) 13.

[20] L. Arzoine, N. Zilberberg, R. Ben-Romano, V. Shoshan-Barmatz, Voltage-dependent anion channel 1-based peptides interact with hexokinase to prevent its anti-apoptotic activity, J. Biol. Chem. 284(6) (2009) 3946-55.

[21] K. Ehrlich, K. Ida, R. Mahlapuu, C. Kairane, I. Oit, M. Zilmer, U. Soomets, Characterization of UPF peptides, members of the glutathione analogues library, on the basis of their effects on oxidative stress-related enzymes, Free Radic. Res. 43(6) (2009) 572-80.

[22] K. Ehrlich, S. Viirlaid, R. Mahlapuu, K. Saar, T. Kullisaar, M. Zilmer, U. Langel, U. Soomets, Design, synthesis and properties of novel powerful antioxidants, glutathione analogues, Free Radic. Res. 41(7) (2007) 779-87.

[23] C.P. Cerrato, U. Langel, Effect of a Fusion Peptide by Covalent Conjugation of a Mitochondrial Cell-Penetrating Peptide and a Glutathione Analog Peptide, Mol Ther Methods Clin Dev 5 (2017) 221-231.

[24] C.P. Cerrato, K. Kunnapuu, U. Langel, Cell-penetrating peptides with intracellular organelle targeting, Expert Opin Drug Deliv 14(2) (2017) 245-255.

[25] L. Ounpuu, L. Truu, I. Shevchuk, V. Chekulayev, A. Klepinin, A. Koit, K. Tepp, M. Puurand, E. Rebane-Klemm, T. Kaambre, Comparative analysis of the bioenergetics of human adenocarcinoma Caco-2 cell line and postoperative tissue samples from colorectal cancer patients, Biochem. Cell Biol. (2018) 1-10.

[26] Y. Zhou, F. Tozzi, J. Chen, F. Fan, L. Xia, J. Wang, G. Gao, A. Zhang, X. Xia, H. Brasher, W. Widger, L.M. Ellis, Z. Weihua, Intracellular ATP Levels Are a Pivotal Determinant of Chemoresistance in Colon Cancer Cells, Cancer Res. 72(1) (2012) 304-314.

[27] S. Shapira, A. Fokra, N. Arber, S. Kraus, Peptides for diagnosis and treatment of colorectal cancer, Curr. Med. Chem. 21(21) (2014) 2410-6.

[28] A.V. Kuznetsov, V. Veksler, F.N. Gellerich, V. Saks, R. Margreiter, W.S. Kunz, Analysis of mitochondrial function in situ in permeabilized muscle fibers, tissues and cells, Nat Protoc 3(6) (2008) 965-76. 
[29] E. Gnaiger, Oxygen Solubility in Experimental Media, OROBOROS Bioenergetics, Newsletter MiPNet 6.3, Innsbruck, Austria, 2001.

[30] M. Puurand, K. Tepp, A. Klepinin, L. Klepinina, I. Shevchuk, T. Kaambre, Intracellular Energy-Transfer Networks and High-Resolution Respirometry: A Convenient Approach for Studying Their Function, Int J Mol Sci 19(10) (2018).

[31] C.P. Cerrato, M. Pirisinu, E.N. Vlachos, U. Langel, Novel cell-penetrating peptide targeting mitochondria, FASEB J. 29(11) (2015) 4589-99.

[32] M. Lonneux, FDG-PET and PET/CT in Colorectal Cancer, PET Clinics 3(2) (2008) 147-153.

[33] F. Graziano, A. Ruzzo, E. Giacomini, T. Ricciardi, G. Aprile, F. Loupakis, P. Lorenzini, E. Ongaro, F. Zoratto, V. Catalano, D. Sarti, E. Rulli, C. Cremolini, M. De Nictolis, G. De Maglio, A. Falcone, G. Fiorentini, M. Magnani, Glycolysis gene expression analysis and selective metabolic advantage in the clinical progression of colorectal cancer, Pharmacogenomics J 17(3) (2017) 258264.

[34] B. Altenberg, K.O. Greulich, Genes of glycolysis are ubiquitously overexpressed in 24 cancer classes, Genomics 84(6) (2004) 1014-20.

[35] O.H. Minchenko, A. Ochiai, I.L. Opentanova, T. Ogura, D.O. Minchenko, J. Caro, S.V. Komisarenko, H. Esumi, Overexpression of 6-phosphofructo-2-kinase/fructose-2,6-bisphosphatase4 in the human breast and colon malignant tumors, Biochimie 87(11) (2005) 1005-10.

[36] P. Dzeja, A. Terzic, Adenylate kinase and AMP signaling networks: Metabolic monitoring, signal communication and body energy sensing, Int J Mol Sci 10(4) (2009) 1729-72.

[37] Y. Ji, C. Yang, Z. Tang, Y. Yang, Y. Tian, H. Yao, X. Zhu, Z. Zhang, J. Ji, X. Zheng, Adenylate kinase hCINAP determines self-renewal of colorectal cancer stem cells by facilitating LDHA phosphorylation, Nat Commun 8 (2017) 15308.

[38] A. Klepinin, L. Ounpuu, R. Guzun, V. Chekulayev, N. Timohhina, K. Tepp, I. Shevchuk, U. Schlattner, T. Kaambre, Simple oxygraphic analysis for the presence of adenylate kinase 1 and 2 in normal and tumor cells, J. Bioenerg. Biomembr. 48(5) (2016) 531-548.

[39] D. Pucar, P.P. Dzeja, P. Bast, R.J. Gumina, C. Drahl, L. Lim, N. Juranic, S. Macura, A. Terzic, Mapping hypoxia-induced bioenergetic rearrangements and metabolic signaling by $18 \mathrm{O}$-assisted 31P NMR and 1H NMR spectroscopy, Mol. Cell. Biochem. 256-257(1-2) (2004) 281-9.

[40] Y.-H. Jan, T.-C. Lai, C.-J. Yang, Y.-F. Lin, M.-S. Huang, M. Hsiao, Adenylate kinase-4 modulates oxidative stress and stabilizes HIF-1 $\alpha$ to drive lung cancer metastasis, bioRxiv (2017).

[41] P. Moreno-Acosta, S. Carrillo, O. Gamboa, A. Romero-Rojas, J. Acosta, M. Molano, J. BalartSerra, M. Cotes, C. Rancoule, N. Magne, Novel predictive biomarkers for cervical cancer prognosis, Mol Clin Oncol 5(6) (2016) 792-796.

[42] Y. Liu, T. Murray-Stewart, R.A. Casero, Jr., I. Kagiampakis, L. Jin, J. Zhang, H. Wang, Q. Che, H. Tong, J. Ke, F. Jiang, F. Wang, X. Wan, Targeting hexokinase 2 inhibition promotes radiosensitization in HPV16 E7-induced cervical cancer and suppresses tumor growth, Int. J. Oncol. 50(6) (2017) 2011-2023.

[43] A. Koit, I. Shevchuk, L. Ounpuu, A. Klepinin, V. Chekulayev, N. Timohhina, K. Tepp, M. Puurand, L. Truu, K. Heck, V. Valvere, R. Guzun, T. Kaambre, Mitochondrial Respiration in 
Human Colorectal and Breast Cancer Clinical Material Is Regulated Differently, Oxid Med Cell Longev 2017 (2017) 1372640.

[44] W. Sperl, D. Skladal, E. Gnaiger, M. Wyss, U. Mayr, J. Hager, F.N. Gellerich, High resolution respirometry of permeabilized skeletal muscle fibers in the diagnosis of neuromuscular disorders, Mol. Cell. Biochem. 174(1-2) (1997) 71-8.

[45] J.F. Turrens, Mitochondrial formation of reactive oxygen species, J Physiol 552(Pt 2) (2003) $335-44$.

[46] M. Redza-Dutordoir, D.A. Averill-Bates, Activation of apoptosis signalling pathways by reactive oxygen species, Biochimica et Biophysica Acta (BBA) - Molecular Cell Research 1863(12) (2016) 2977-2992.

[47] P.E. Porporato, V.L. Payen, J. Perez-Escuredo, C.J. De Saedeleer, P. Danhier, T. Copetti, S. Dhup, M. Tardy, T. Vazeille, C. Bouzin, O. Feron, C. Michiels, B. Gallez, P. Sonveaux, A mitochondrial switch promotes tumor metastasis, Cell Rep 8(3) (2014) 754-66.

[48] S. Rodic, M.D. Vincent, Reactive Oxygen Species (ROS) are a key determinant of cancer's metabolic phenotype, Int. J. Cancer 142 (2018) 440-448.

[49] D. Pesta, E. Gnaiger, High-Resolution Respirometry: OXPHOS Protocols for Human Cells and Permeabilized Fibers from Small Biopsies of Human Muscle

Mitochondrial Bioenergetics, in: C.M. Palmeira, A.J. Moreno (Eds.), Humana Press2012, pp. 2558.

[50] F. Sotgia, M.P. Lisanti, Mitochondrial markers predict survival and progression in non-small cell lung cancer (NSCLC) patients: Use as companion diagnostics, Oncotarget 8(40) (2017) 6809568107.

[51] J. Neuzil, J.C. Dyason, R. Freeman, L.F. Dong, L. Prochazka, X.F. Wang, I. Scheffler, S.J. Ralph, Mitocans as anti-cancer agents targeting mitochondria: lessons from studies with vitamin $\mathrm{E}$ analogues, inhibitors of complex II, J. Bioenerg. Biomembr. 39(1) (2007) 65-72. 